\title{
Synthetic Spectra of Magnetic White Dwarfs
}

\author{
Stefan Jordan \\ Institut für Theoretische Physik und Sternwarte der Universität Kiel \\ Olshausenstraße 40, D-2300 Kiel 1, Federal Republic of Germany
}

\begin{abstract}
A new program for the calculation of theoretical spectra and wavelength dependend degrees of the linear and circular polarization for magnetic white dwarfs with hydrogen atmospheres has been developed. The main improvement compared to previous models is a more realistic treatment of the bound-free absorption and of the magnetooptical parameters arising from free electrons in the continuum. For the newly discovered magnetic white dwarf HS $1254+3430$ a polar field strength of $18 \mathrm{MG}$ was found. Calculations for the high field object $\mathrm{Grw}+70^{\circ} 8247$ confirms the results of Wickramasinghe and Ferrario $(320 \mathrm{MG})$ but gave a better fit to the spectrum at wavelengths larger than $6200 \AA$.
\end{abstract}

\section{Introduction}

From the position of the Zeeman components in the spectrum of magnetic white dwarfs approximate field strenths can be determined. To get more infomation about the field structure detailed computer models are necessary. Martin and Wickramasinghe (see Wickramasinghe and Ferrario, 1988 for references) analyzed four DA white dwarfs between 5 and 36 MG by solving the equations of radiative transport in a magnetized atmosphere and using the Zeeman calculations of Kemic (1974). Since 1984 line data for $B \gtrsim 10^{8} \mathrm{G}$ are available (Rösner et al., 1984 and Forster et al., 1984, Tübingen; Henry and O'Connell, 1984) and made the interpretation of objects with very strong fields possible. Assuming a dipol with a polar field strength of $B_{p}=320 \mathrm{MG}$ Wickramasinghe and Ferrario (1988, referred to as WF) were able to get a good fit to the spectrum of the famous object $\mathrm{Grw}+70^{\circ} 8247$ between 3500 and $6200 \AA$. The calculated fux at longer wavelengths and the prediction for the linear and circular polarization showed major discrepancies with the observations made by Angel et al. (1985).

New synthetic spectra have been computed with the program described in this paper. They confirm the field configuration of Grw $+70^{\circ} 8247$ found by WF but give a better agreement with the observed flux between 6200 and $9200 \AA$. Since polarization is more sensitive to changes in the continuum opacities than the flux, the calculated polarization still differs seriously from the observed values.

\section{Model Description}

Special attention was given to the physics at fields higher than $100 \mathrm{MG}$. The details of the model will be described in a forthcoming paper. Here only a short synopsis can be given:

- Zero field pure hydrogen model atmospheres (Koester et al, 1979) for $\log g=8$ have been used to determine the temperature and pressure stratification. This can only be justified if Lorentz forces do not dominate the hydrostatic structure at optical depths $\gtrsim 10^{-2}$. Provided that the decay of the global magnetic field of the star is responsible for the electric current distribution in the outer layers - only slow rotation is allowed in this case rotating - a procedure of Landstreet (1987) can be applied to estimate the strength of the Lorentz forces. With the new data of Wendell et 
al. (1987) for the decay time of the field, which differ by up to a factor of eight from the values of Fontaine et al. (1973) used by Landstreet, it can be shown that Lorentz forces are negligible even at fields higher than $100 \mathrm{MG}$.

Since the measured degree of polarization normally amounts only to a few percent $(<7 \%$ in Grw $+70^{\circ} 8247$ ) the normal equation of radiative transport is sufficient for the model atmosphere.

A test calculation indicates that cyclotron absorption can become important at $B>100 \mathrm{MG}$ and lead to somewhat smaller temperatures at small optical depths compared to the models that are the basis for the spectra of $\mathrm{Grw}+70^{\circ} 8247$ presented in this paper.

- The stellar surface is devided into a grid of small elements by circles of constant latitude and longitude (the magnetic pole defines the orientation of the spherical coordinate system). After a field configuration (e.g. a centered dipol) is specified the average vector and the variation of the magnetic field is determined for each element.

- On each surface element the coupled equations of radiative transport in a magnetized plasma (Hardorp et al., 1976) is solved for the Stokes parameters $I, Q, U$ and $V$ by a method similar to that of Martin und Wickramasinghe (1979). The results must be integrated in order to get the emergent flux and polarization for the whole stellar disc.

- New tables from the Tübingen group (by courtesy of Wunner, 1986) - containing a narrower grid of line positions and opacities than the published data - make it possible to take quick oscillations of the line strengths with varying magnetic field (Wunner et al, 1986) into account.

- While exact data for the lines are available the situation concerning the continuum opacities is very unsatisfactory. The first order approximation of Lamb and Sutherland (1974) is still the only existing method to calculate hydrogen bound-free absorption for different changes of the magnetic quantum number $\Delta m$. The procedure calculates the oscillator strengths from zero field values at a frequency shifted by the Larmor value $\left(e B / 4 \pi m_{e} c\right) \Delta m$. As one can see from the asymptotic behaviour of the line components the position of the absorption edges can not be described by this simple Zeeman splitting even at arbitrarily small fields. To get correct wavelengths for the boundfree threshold the energy difference between the exactly known bound states and the minimum energy of the lowest Landau level that can be reached by a specific $\Delta m$ transitions is used here. At the Balmer edge 12 transitions ( 4 for each $\Delta m$ ) with 8 different wavelenths are found (see figure 1 ). The Lamb and Sutherland opacities are distributed to the different $\Delta m$ components by applying the Wigner Eckart theorem.

While thus the begin of the bound-free absorption is known, problems have to be expected if the Lamb and Sutherland approximation is used for the calculation of the absorption strengths at $B \gtrsim 5 \cdot 10^{7} \mathrm{G}$.

\section{Figure 1:}

bound-free transitions at the Balmer edge with minimum energy difference.

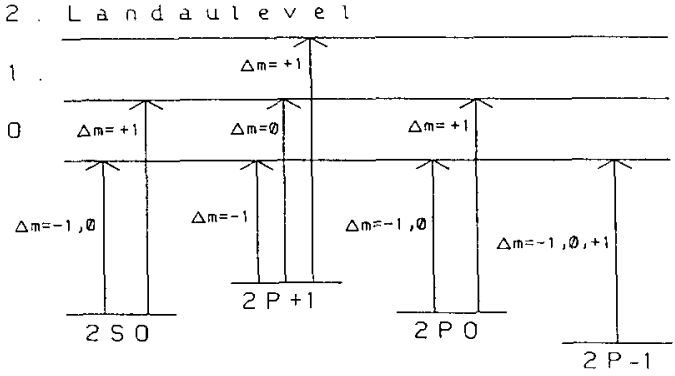

- Cyclotron absorption is included allowing for simultaneous broadening of the absorption profile by the Doppler effect and collisions of electrons.

- The magnetooptical parameters of the lines are calculated following a method of Wittmann (1974). The formulae of Pacholczyk (1976) have been used by Martin und Wickramasinghe (1982) to calculate the Faraday and Voigt effect arising from free electrons in the continuum. The singularity at the cyclotron frequency in this formulae is avoided here by using a consistent approach (Jordan and O'Connell, 1987) that takes electron collisions into account. 
- The profiles of the opacities and the magnetooptical parameters are broadened by the magnetic field variation over a single surface element. This minimizes numerical bumps resulting from the discretization of the stellar disc and the number of surface elements neccessary for the computation.

\section{Results}

The program was tested by calculating synthetic spectra for the 36 MG object BPM 25114 which has already been analyzed by Martin und Wickramasinghe (1978). The agreement with the observation is somewhat better in detail mainly because of the new line opacities used here.

The observed spectrum of HS $1254+3430$ was compared to theoretical spectra for models with different field configurations and effective temperatures. A good agreement was found for $B_{p}=18 \pm 2 \mathrm{MG}$, a viewing angle with respect to the dipol axis of $i=70^{\circ} \pm 20^{\circ}$ and $T_{\text {eff }}=$ $14000 \pm 2000 \mathrm{~K}$ (see figure 2).

Figure 2:

Synthetic spectrum for $T_{e f f}=15000 K$,

$B_{p}=18 \mathrm{MG}$ and $i=70^{\circ}$

compared to the observa-

tion of HS $1254+3430$

(Hagen et al., 1987).

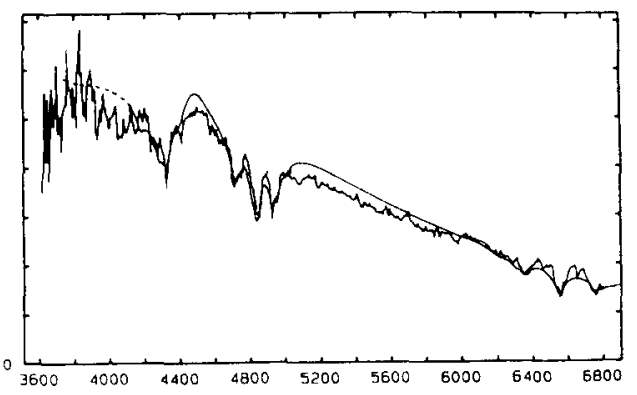

Grw $+70^{\circ} 8247$ has a much stronger field strength. Figure 3 (top) compares a synthetic spectrum for $T_{\text {eff }}=16000 \mathrm{~K}$ with the observations of Angel et al. (1985, the two center curves) and confirms the result of WF (bottom, $T_{e f f}=14000 \mathrm{~K}$ ) who found a dipol field with $B_{p}=320 \mathrm{MG}$ and $i=30^{\circ}$.

\section{Figure 3:}

Spectra of

Grw $+70^{\circ} 8247$ (see text for explanations)

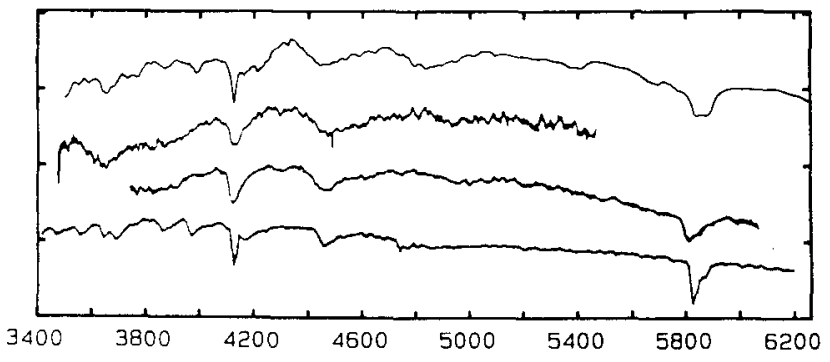

While WF were not able to obtain a good fit in the red and infrared region (figure 4a) figure 4b gives a reasonable agreement with the observed flux distribution.

The absolute strength and the wavelength dependence of the linear and circular polarization predicted by the model used here still differs seriously from the observations and shows no improvement compared to the results of WF.

While the flux calculations suggest that the total continuum opacity is fairly good reproduced by the new method, the contributions of the different $\Delta m$ transitions are not correctly described by the Lamb and Sutherland approximation at strong fields. Further progress can therefore only be expected when consistend multichannel calculations for the continuum absorption become available. 
Figure 4a:

Synthetical spectrum of $\mathrm{Grw}+70^{\circ} 8247$

at long wavelengths (Wick-

ramasinghe and

Ferrario, 1988)

Figure 4b:

New fit

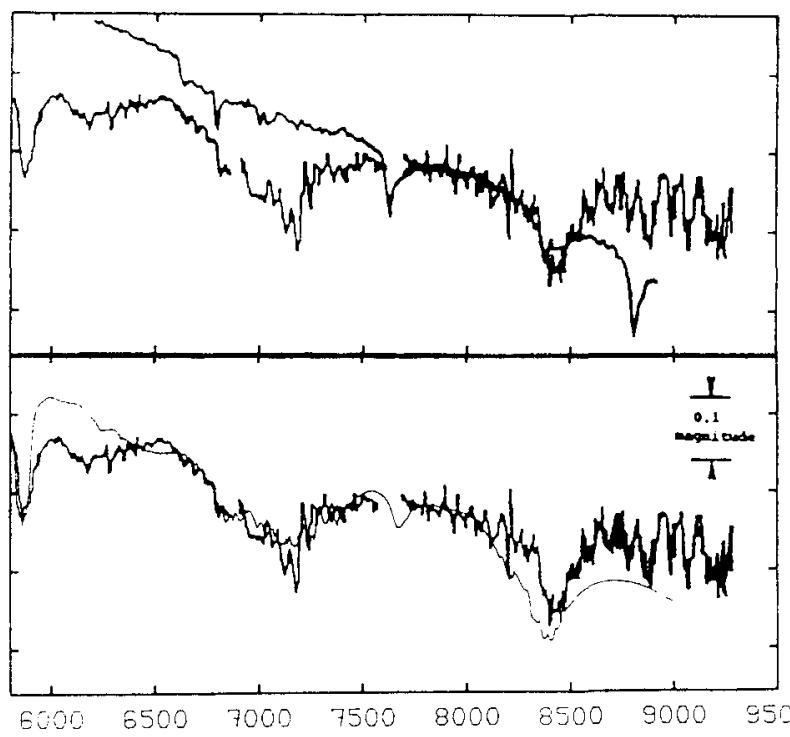

References

Angel, J.R.P., Liebert, J. \& Stockman, H.S., 1985: Astrophys. J. 292, 260

Fontaine, G., Thomas, J.H. \& Van Horn, H.M. ,1973: Astrophys. J. 184, 911

Forster, H., Strupat, W., Rösner, W., Wunner, G., Ruder, H. \& Herold, H., 1984: J. Phys. B: AT. Mol. Phys. 17, 1301

Hagen, H.-J., Groote, D., Engels, D., Haug, U., Toussaint, F. \& Reimers, D., 1987:

Astron. Astrophys. 183, L7

Hardorp, J., Shore, S.N. \& Wittmann, A., 1976: Physics of $A_{p}$ stars, IAU-Colloquium No. 32, 41

Kemic, S.B., 1974: Joint Inst. Lab. Astrophys. Rep. No. 113

Jordan, S.H. \& O'Connell, R.F., 1987: unpublished

Koester, D., Schulz, H. \& Weidemann, V., 1979: Astron. Astrophys. 76, 262

Landstreet, J.D., 1986: Mon. Not. R. astr. Soc., 225, 437

Martin, B. \& Wickramasinghe, D.T., 1978: Mon. Not. R. astr. Soc. 189, 883

Martin, B. \& Wickramasinghe, D.T., 1979: Mon. Not. R. astr. Soc. 183, 533

Martin, B. \& Wickramasinghe, D.T., 1982: Mon. Not. R. astr. Soc. 200, 993

Pacholczyk, A.G., 1976: Radio Galaxies, Pergamon Press, London

Rösner, W., Wunner, G., Herold, H. \& Ruder, H., 1984: J. Phys. B: At. Mol. Phys. 17, 29

Wendell, C.E. \& Van Horn, H.M., 1987: Astrophys. J. 313, 284

Wickramasinghe, D.T. \& Martin, B. , 1986: Mon. Not. R. astr. Soc. 223, 323

Wickramasinghe, D.T. \& Ferrario, L., 1988: Astrophys. J. 327, 222

Wittmann, A., 1974: Sol. Phys. 35, 11

Wunner, G., Rösner, W., Herold, H. \& Ruder, H., 1985: Astr. Astrophys. 149, 102 